\title{
Cadaveric Donor Factor Variations During a 12-Year Period: Influence on Kidney Transplant Outcomes
}

\author{
P. Nunes, B. Parada, J. Pratas, A. Roseiro, A. Figueiredo, F. Macário, F. Rolo, and A. Mota
}

\begin{abstract}
Our purpose was to evaluate changes in cadaveric donor factors between 1993 and 2004 and their impact on the short- and long-term outcomes of renal transplants in a single center.
\end{abstract}

Patients and methods. Cadaveric renal transplants performed in our unit between 1993 and 2004 were divided in two groups of identical length: A $(n=455 ; 1993-1998)$ and B ( $n=465 ; 1999-2004)$. Major differences related to donor, graft, and recipient factors were analyzed between groups and correlated with main outcome parameters. Recipient age, gender, weight, etiology of end-stage renal disease, average length of dialysis, and cold ischemia were not different in the two periods.

Results. Grafts harvested in our hospital were more frequent in group A (92.3 vs $78.2 \% ; P<.005)$. Traumatic causes of death were more frequent before 1999: 90.9 vs $70.9 \%(P<.001)$. Mean donor age was higher after 1999: 31.37 vs 35.94 years $(P<$ $.005)$. Female donors were more frequent in the second period: 20.5 vs $26.6 \%(P<$ $.05)$. Mean donor weight was also higher: $52.36 \mathrm{vs} 67.86 \mathrm{~kg}(P<.05)$. All of these differences were unfavourable characteristics regarding graft outcomes. Delayed graft function $(\mathrm{A}=13 \%, \mathrm{~B}=24.2 \%)$, acute rejection episodes $(\mathrm{A}=41.2 \%, \mathrm{~B}=28 \%)$, and chronic allograft dysfunction $(\mathrm{A}=23.5 \%, \mathrm{~B}=14.4 \%)$ were also significantly different between the two cohorts $(P<.005)$. Graft function (serum creatinine at 1 and 2 years), patient and graft survivals, causes of graft loss, and of patient death were similar across time.

Conclusion. The unfavorable tendency in the quality of cadaveric donors during the last 12 years had no negative impact on graft function and patient outcome.

$\mathrm{T}$ HE KEY IMPORTANCE of donor factors in the short- and long-term performance of kidney grafts is well known. ${ }^{1,2}$ Young healthy male donors who died from traumatic causes in a hospital near the transplantation department are progressively rare. ${ }^{3}$ However, the rising figures of patients awaiting kidney transplantation and the scarcity of organs has lead to an increasing use of cadaveric grafts once thought inadequate.

This study sought to explore the alterations in cadaveric donor factors during the last years, disclosing its impact on kidney transplant outcomes at a single center.

\section{PATIENTS AND METHODS}

All 920 cadaveric kidney transplantations performed in our department between January 1993 and December 2004 were included in this retrospective analysis. They were divided into two groups covering equal time spans of 6 years: group A $(n=455 ; 1993-1998)$ versus group B $(n=465 ; 1999-2004)$. We calculated for each group the main factors related to the donor, graft, and recipient in terms of outcome measures for comparisons.

The statistical analysis was performed using SPSS for Windows 10.0. Categorical metrics were compared by chi square-testing, and continuous variables by Student's $t$ test, with Yates's correction or Fisher's exact test wherever appropriate. Graft and patient cumulative actuarial and actual survival curves were examined by Kaplan-Meier analysis with differences assessed with the Mantel-

From the Department of Urology and Renal Transplantation, Hospitais da Universidade de Coimbra, Coimbra, Portugal.

Address reprint requests to Pedro Nunes, Department of Urology and Renal Transplantation, Hospitais da Universidade de Coimbra, 3000 Coimbra, Portugal. E-mail: ptnunes@ oniduo.pt

0041-1345/06/\$-see front matter doi:10.1016/j.transproceed.2006.06.058 
Cox log-rank test. $P<.05$ was considered significant in every test for differences.

\section{RESULTS}

There were no significant differences $(P=$ NS) between the two groups regarding recipient mean age $(A=42.17$ vs $\mathrm{B}=42.22$ years); male gender (64.8 vs $68.8 \%)$; mean body weight ( $63.03 \mathrm{vs} 64.04 \mathrm{~kg}$ ); comorbidities; etiology of end-stage renal disease; or average time on previous renal replacement therapy. Surgical procedures were basically the same over time. HLA compatibilities (A, B, DR) were greater during the second period $(\mathrm{A}=2.18$ vs $\mathrm{B}=$ $2.81, P<.005)$. Initial immunosuppressive schemes showed significant differences reflecting the introduction of new agents.

Mean donor age $(\mathrm{A}=31.37$ vs $\mathrm{B}=35.94$ years $)$ and the proportion of donors older than 60 years $(\mathrm{A}=3.5 \%$ vs $\mathrm{B}=$ $11.2 \%$ ) were significantly different between the two periods $(P<.05)$. The proportion of female donors was higher in the second period $(\mathrm{A}=20.5$ vs $\mathrm{B}=26.6 \%, P<.05)$. Mean body weight was higher in group B $(52.36$ vs $67.86 \mathrm{~kg}, P<$ $.005)$, but mean serum creatinine was lower (1.14 vs 1.00 $\mathrm{mg} / \mathrm{dL}, P<.005)$.

The proportion of grafts harvested outside our hospital increased after 1999 ( $\mathrm{A}=7.69$ vs $\mathrm{B}=21.75 \%, P<.005)$, but mean cold ischemia time remained similar $(\mathrm{A}=20.88$ vs $\mathrm{B}=20.65$ hours, $P=\mathrm{NS}$ ). Multiple-organ versus kidney-only procurement procedures were more common in group B $(74.07 \%$ vs $86.55 \%, P<.005)$. Traumatic events as the cause of donor death were less common in the last 6 years $(\mathrm{A}=90.9$ vs $\mathrm{B}=70.9 \%, P<.005)$.

The donor age group $(<60$ vs $>60)$ affected the percentage of delayed graft function (DGF; 17.5 vs $36.7 \%, P<$ .001 ), of acute rejection (AR) episodes (33.1 vs $52.5 \%$; $P<.001)$ and of chronic allograft dysfunction $(21.5 \mathrm{vs}$ $51.9 \%, P<.001)$ as well as graft half-life $(9.05$ vs 7.08 years, $P<.005)$. In our series, female donor gender was associated with a higher probability of DGF (17 vs $23 \%, P<$ $.005)$. Local graft source was associated with a lower probability of DGF (13.2 vs $38.1 \%, P<.005)$. Cause of death (traumatic vs other) influenced the percentage of DGF (13.9 vs $38.6 \%, P<.001)$ and of chronic allograft dysfunction $(21.52$ vs $35.1 \%, P<.001)$ as well as graft half-life $(9.21$ vs 7.60 years, $P<.001)$.

Mean follow-up was 6.77 and 2.64 years for groups A and $\mathrm{B}$, respectively $(P<.005)$. DGF was significantly higher after 1999 ( $\mathrm{A}=12.95$ vs $\mathrm{B}=24.24 \%, P<.005)$, but the percentage of patients showing AR was lower $(\mathrm{A}=41 \%$ vs $\mathrm{B}=28 \%, P<.005)$. Chronic allograft dysfunction was higher in group A $(23.5$ vs $14.44 \%, P<.005)$. Mean serum creatinine at 1 month was significantly higher among group B $(1.65$ vs $1.85 \mathrm{mg} / \mathrm{dL}, P<.005)$, but there were no differences at 1 and 2 years. Actuarial cumulative graft survival at 1 and 5 years was $90.1 \%$ and $76.9 \%$ for group A and $91.2 \%$ and $82.5 \%$ for group B (log rank 0.098). Causes of graft loss did not differ between two groups. Actuarial cumulative patient survival at 1 and 5 years was $93.3 \%$ and
87.1\% for group A and $96.59 \%$ and $89.9 \%$ for group B (log rank 0.135). Death causes were similar.

\section{DISCUSSION}

When kidney transplantation was not widely available and waiting lists were short, the majority of units selected grafts from the best cadaveric donors. For the medically suitable end-stage renal disease patient, transplantation is the therapeutic modality that provides the best length and quality of life. ${ }^{4}$ The enlarged waiting lists, leading to a shortage of cadaveric organs, has decreased the chance to choose only top-quality donors. ${ }^{3}$ The ideal deceased donor is a young person who dies from isolated traumatic head injury with no thoracic or abdominal organ function involvement. Such a deceased donor provides excellent transplantable organs with an opportunity to achieve immediate allograft function and long-term patient survival. ${ }^{5}$

During the last years we have seen a progressive rise in the proportion of older donors with nontraumatic causes of death. Donor age is one of the best identified prognostic factors in renal transplantation. ${ }^{6}$ In our experience, donor age over 60 years is related to a higher probability of DGF, AR, chronic dysfunction and shorter graft half-life. The proportion of females, another well-defined prognostic factor, ${ }^{7}$ was greater during the last period in our study. In our unit, delayed graft function was more common using female grafts.

The progressive increment in the percentage of organs harvested outside our hospital, was identified in our series as a risk factor for DGF despite not increasing cold ischemia. ${ }^{8}$ We gradually engrafted more organs from cadaveric donors who died of nontraumatic causes, mainly from cerebral vascular events. Our analysis confirmed that the best results were obtained when the cause of death was traumatic (largely cranial trauma from traffic accidents): there was a lower incidence of DGF and chronic allograft dysfunction as well as longer graft half-life. ${ }^{2,5}$

This unfavorable evolution of the major prognostic factors, dependent on donor characteristics, has led to a greater incidence of DGF. Graft function was poorer just in the short-term (1 month) but graft and patient survivals were better in the second period. Furthermore, AR was less common after 1999. The second time period showed a lower incidence of chronic allograft dysfunction; although it also had a shorter follow-up. Some possible explanations include increased team experience managing transplant patients (selection and preparation of donors and recipients, harvesting, and transplantation surgical procedures, handling of surgical and medical complications) and new safer, more powerful immunosuppressive agents.

\section{REFERENCES}

1. Swanson SJ, Hypolite IO, Agodoa LY, et al: Effect of donor factors on early graft survival in adult cadaveric renal transplantation. Am J Transplant 2:68, 2002 
2. Pessione F, Cohen S, Durand D, et al: Multivariate analysis of donor risk factors for graft survival in kidney transplantation. Transplantation 75:361, 2003

3. Miranda B, Canon J, Naya MT, et al: Kidney donor profile in Spain: risks factors and characteristics of the organs rejected for transplantation. Ann Transplant 8:9, 2003

4. Wolfe RA, Ashby VB, Milford EL, et al: Comparison of mortality in all patients on dialysis, patients on dialysis awaiting transplantation, and recipients of a first cadaveric transplant. N Engl J Med 341:1725, 1999
5. Metzger RA, Delmonico FL, Feng S, et al: Expanded criteria donors for kidney transplantation. Am J Transplant 3(suppl 4):114, 2003

6. Oppenheimer F, Aljama P, Asensio Peinado C, et al: The impact of donor age on the results of renal transplantation. Nephrol Dial Transplant 19(suppl 3):iii11, 2004

7. Zeier M, Dohler B, Opelz G, et al: The effect of donor gender on graft survival. J Am Soc Nephrol 13:2570, 2002

8. Roodnat JI, Mulder PG, Van Riemsdijk IC, et al: Ischemia times and donor serum creatinine in relation to renal graft failure. Transplantation 75:799, 2003 